\title{
Recherches en terrain difficile : le cas du culte traditionnel annobonais (île d'Annobon, Guinée équatoriale, $\mathrm{XV}^{\mathrm{e}}-\mathrm{XX}^{\mathrm{e}}$ siècle)
}

Research in Difficult Terrain: The Case of Traditional Annobonese Worship

(Annobon Island, Equatorial Guinea, 15th-20th century)

\section{Valérie de Wulf}

\section{OpenEdition}

Journals

Édition électronique

URL : https://journals.openedition.org/etudesafricaines/25253

DOI : $10.4000 /$ etudesafricaines.25253

ISSN : $1777-5353$

Éditeur

Éditions de l'EHESS

\section{Édition imprimée}

Date de publication : 14 mars 2019

Pagination : 171-191

ISSN : 0008-0055

Référence électronique

Valérie de Wulf, "Recherches en terrain difficile : le cas du culte traditionnel annobonais (île d'Annobon, Guinée équatoriale, XVe-XXe siècle) », Cahiers d'études africaines [En ligne], 233 | 2019, mis en ligne le 14 mars 2021, consulté le 08 janvier 2022. URL : http://journals.openedition.org/ etudesafricaines/25253; DOI : https://doi.org/10.4000/etudesafricaines.25253 


\title{
Recherches en terrain difficile
}

\author{
Le cas du culte traditionnel annobonais \\ (île d'Annobon, Guinée équatoriale, $X V^{e}-X X^{e}$ siècle)
}

En France, la Guinée équatoriale est peu connue. Certes, c'est un petit pays, sa taille est proche de celle de la Belgique, mais cette méconnaissance est principalement due à son histoire. Bien que localisée entre deux anciennes colonies de l'Afrique équatoriale française, le Cameroun et le Gabon, la Guinée équatoriale était une colonie espagnole jusqu'en 1968, date de son indépendance. Au-delà de son histoire, cet oubli est aussi dû à sa situation post-coloniale : dès 1969, son premier président élu démocratiquement organisa un putsch et se déclara peu de temps après président à vie. Même si ce dictateur est aujourd'hui décédé, la dictature a profondément marqué le pays et ses institutions. La police et l'armée sont omniprésentes. Leurs agents sont en majorité illettrés et ne comprennent pas l'utilité des recherches en sciences sociales. Ils les assimilent, le plus souvent, à une forme d'espionnage. De ce fait, les travaux de recherche menés sur place sont régulièrement entravés ou interrompus. Des efforts ont pourtant été consentis par le gouvernement actuel, afin de rendre le pays plus attractif: il est devenu facile d'obtenir un visa et des infrastructures ont été mises en place pour désenclaver le pays aussi bien dans sa partie continentale qu'insulaire. Mais le régime sécuritaire en place, et les difficultés rencontrées sur le terrain, sont encore trop importantes pour que les chercheurs étrangers soient nombreux à prendre le risque de s'investir dans l'étude de ce pays et de ses populations ${ }^{1}$.

Alertée de ces handicaps, mais tentée malgré cela de me consacrer à l'étude de ce pays, où tant reste encore à découvrir, j'ai dès que possible complété les

1. Fort heureusement, des étudiants équato-guinéens formés à l'université nationale ont commencé à travailler sur leur histoire, leurs coutumes et leurs traditions, mais leurs opportunités de publication restent très limitées : dans ce pays, il n'existe ni imprimerie, ni édition, une seule librairie de dépôt, et un nombre restreint de bibliothèques. 
manques de mes séjours en Guinée équatoriale en 1996, en 2011 et en 2015 par des données historiques et anthropologiques, plus aisées à rassembler en Europe. C'est ainsi qu'en dehors des rencontres que j'ai pu faire en Espagne en 1997 et en 2013, où une partie de la diaspora a trouvé refuge, notamment les membres de l'association annobonaise Viyil, je me suis attelée à rechercher des documents d'archives et des publications attestant de l'histoire de l'île et de ses habitants sur le temps long.

Découverte par les Portugais à la fin du $\mathrm{XV}^{\mathrm{e}}$ siècle, Annobon a été une colonie des souverains lusitaniens officiellement jusqu'à la fin du XVIII ${ }^{\mathrm{e}}$ siècle. Je me suis rendue à Lisbonne dans les centres d'archives susceptibles de posséder la documentation la plus fournie sur l'île et ses habitants. C'est ainsi que j'ai trouvé des documents très intéressants principalement à l'Arquivo Nacional de Torre do Tombo (ANTT il y a quelques années, TT à présent), à l'Arquivo Historico Ultramarino (AHU) et à la Biblioteca Nacional (BNL). La piste que j'ai suivie ensuite est celle de l'Espagne, puisque l'île a été échangée officiellement à la fin du XVIII ${ }^{\mathrm{e}}$ siècle, au profit de ce pays. Pour cela, j'ai consulté les archives du Museo Naval de Madrid (MN), l'Archivo General de Simancas (AGS) et l'Archivo General de Indias à Séville (AGI).

La colonisation espagnole n'a été réellement effective dans l'île qu'à la fin du XIX ${ }^{e}$ siècle. Elle se concrétise avec l'installation de la mission des clarétains, de l'ordre du Cœur Immaculé de Marie, choisi par la couronne espagnole pour évangéliser le peuple équatoguinéen. Ces missionnaires se mettent, à l'exemple des jésuites, à compiler tout ce qu'ils peuvent observer de la population, de ses croyances et de ses rituels. Nombreux sont aussi les rapports qu'ils envoient au gouvernement colonial dont ils sont les seuls représentants officiels dans l'île jusqu'en 1906, date à partir de laquelle un délégué nommé par le gouvernement va encadrer la population à leurs côtés. Pour en savoir plus sur cette période, je fais de nombreuses recherches à l'Archivo General de la Administración Civil del Estado de Alcala de Henarés (AGA), et j'ai dépouillé différentes archives missionnaires : outre celles de l'ordre des clarétains à Vic en Espagne et à Rome, j'ai cherché dans les documents rédigés par les jésuites espagnols qui ont précédé les clarétains en Guinée au milieu du XIX ${ }^{\mathrm{e}}$ siècle. J'y ai aussi recueilli des témoignages écrits divers de religieux qui sont rassemblés dans les archives de la Propaganda Fide (APF).

Parmi les publications existantes, certaines m'ont aussi aidé considérablement, comme par exemple : l'ouvrage de Carlos Agostinho das Neves (1989), intitulé São Tomé e Princípe na Segunda Metade do Século XVIII, ou la fameuse Monumenta Missionaria Africana d'António Brásio (1953)².

2. Voir également les douze volumes de Monumenta Misionaria Africana publiés entre 1952 et 1988 à Lisbonne. 
Viennent ensuite les ouvrages rédigés par les missionnaires clarétains à la fin du XIX et surtout au début du $\mathrm{XX}^{\mathrm{e}}$ siècle : notamment celui de Nathalio Barrena (1965), La Isla de Annobón. Et, enfin, des ouvrages ou manuscrits écrits de la main même des Annobonais qui ont eu le souci de consigner leurs traditions et les souvenirs réunis en écoutant les anciens. Le premier d'entre eux reprend la forme des relations écrites par les missionnaires, il s'agit d'une compilation La Noticia de Annobón (Su Geografiá, Historiá y Costumbres) dont l'auteur n'est autre que le premier instituteur annobonais, Miguel Zamora Loboch (1962). Un autre ouvrage plus atypique est celui de Pedro Bodipo Lisso. Rédigé non plus à la moitié, mais à la fin du $\mathrm{XX}^{\mathrm{e}}$ siècle, alors que l'indépendance de la Guinée équatoriale a fait son œuvre, son auteur n'a pas été empêché par le carcan missionnaire de s'exprimer. De ce fait, son avis sur ce qui l'entoure est plus riche, fouillé et libre dans sa forme et son expression. Le manuscrit qu'il m'avait remis en main propre en 1996, après avoir participé aux entretiens que je menais alors, a été publié en 2015 sous le titre Annobón : su tradición, usos y costumbres (Bodipo Lisso 2015).

C'est grâce à la combinaison de toutes ces informations qu'il est possible aujourd'hui de connaître, dans ses grandes lignes, la genèse du peuple annobonais et, à travers lui, de comprendre et reconstituer les principales composantes de son culte.

La contextualisation géographique et historique de l'île et de sa population me semblant indispensable, cet article commencera par une description de l'île, de son histoire dans le temps, puis seront abordés différents aspects du culte local ainsi que ses acteurs principaux. La conclusion portera sur ce que ce culte a apporté aux insulaires et comment il continue d'être actif dans la communauté.

Annobón, une petite île très isolée

Annobón est une petite île de $18 \mathrm{~km}^{2}$. Elle se trouve dans la ligne volcanique du Mont Cameroun à la suite des îles de Bioko, de Principe et de São Tomé dans le golfe de Guinée. Son isolement est total, elle se situe à plus de $100 \mathrm{~km}$ de l'île de São Tomé, et à environ $350 \mathrm{~km}$ des côtes du Gabon, la terre continentale la plus proche. Malgré cela, sa possession s'est toujours révélée stratégique, tout d'abord par sa position sur les routes commerciales maritimes et, aujourd'hui, parce que le domaine maritime de la Guinée équatoriale s'en trouve fortement agrandi et que son sous-sol contient à grande profondeur d'importantes réserves de pétrole.

L'île apparaît pour la première fois sur les portulans et les cartes marines grâce aux campagnes de grandes découvertes menées par les Portugais au XV ${ }^{\mathrm{e}}$ siècle. Elle est alors déserte (Cortesão 1971 : 12-18). 
Elle devient la propriété d'un capitaine donataire dès $1503^{3}$, mais les différentes tentatives menées pour la peupler et l'exploiter n'aboutissent pas. Résultat de ces échecs, dus notamment au fait que ces projets sont pilotés depuis le Portugal, Luis de Almeida, un noble portugais vivant sur l'île voisine de São Tomé, est autorisé par le roi à acquérir l'île et son titre 4 . Il s'agit d'un cas unique dans le monde portugais outre-atlantique. Mais cet homme n'est pas n'importe qui : il est écuyer de la cour et neveu de Baltasar de Almeida, un riche et célèbre négrier très apprécié de la couronne. De par ses domaines dans l'île de São Tomé, et de son mariage avec une riche héritière métisse originaire de cette même île, il a les moyens physiques et financiers pour suppléer aux besoins des nouveaux habitants d'Annobón qu'il a acheminés dans l'île à partir de $1559^{5}$ (de Wulf 2014, t. 1 : 92).

La population qui vient s'installer à Annobón est principalement constituée de métis et d'Africains libres accompagnés d'esclaves. Ils ont, pour la plupart, séjourné auparavant sur l'île de São Tomé, dont ils parlent la langue. Ils sont en majorité déjà baptisés et instruits dans la foi catholique par les missionnaires. En effet, de la fin du Xve siècle à la moitié du XVI ${ }^{\mathrm{e}}$ siècle, nombreux sont les missionnaires venus évangéliser les insulaires de São Tomé. D'abord des franciscains puis, à partir de 1487, des dominicains (de Alencastro 2006 : 155-160). En 1500, le premier ordre religieux, celui des augustins, est nommé officiellement pour s'établir dans la région (Lourenço Farinha 1942 : 124). Les dominicains prennent leur suite (Caldeira 2007 : 61) et, au début du XVII ${ }^{\mathrm{e}}$ siècle, les jésuites sont à leur tour désignés pour s'installer dans le golfe de Guinée (de Alencastro 2008 : 199).

Lorsque les nouveaux habitants arrivent à Annobón, ils sont accompagnés de missionnaires qui y créent une mission permanente. Louis de Almeida y fait construire une église, acquiert tous les ornements et ustensiles nécessaires au culte (Lourenço Farinha 1942 : 144). Ce capitaine donataire prend cette tâche à cœur car il est très pieux. Il fait partie, avec sa femme, des grands donateurs attachés à l'Église. De plus, ils aident les jeunes Santoméens africains ou métis libres à étudier au Portugal (Ambrosio 1987, 21 : 3-22, 22 : 25-40). Paradoxalement, ils ne remettent pas l'esclavage en cause, ils possèdent d'ailleurs un grand nombre d'esclaves dans leurs domaines, mais ils favorisent leur affranchissement s'ils les jugent méritants.

3. PT/TT, Livro das Ilhas, fol. 126, Lettre datée du 16 octobre, rédigée par le roi Emmanuel I ${ }^{\text {er }}$, dans laquelle il indique qu'il concède l'île à Jorge de Melo.

4. PT/TT, Chancelaria de D. Sebastião, Livro 20, fol. 265-268v.

5. Luis de Almeida utilise, pour mener à bien cette tâche, ses revenus très importants de domaines (des orangeraies) qu'il possède sur l'île voisine de São Tomé. 
Les missionnaires restent ainsi quelques années aux côtés des Annobonais mais, après le décès de ce capitaine donataire, la situation change peu à peu : de la période d'avant les jésuites, c'est-à-dire avant le début du XVII ${ }^{\mathrm{e}}$ siècle, on ne sait pas grand-chose, si ce n'est qu'un prêtre de couleur officie sur place en 1592 (Pujadas 1968 : 427). Une lettre datée de 1597 précise que ce missionnaire n'est plus auprès de la population ${ }^{6}$ (Brásio 1953 : 584-585). À partir de cette date, il n'y a plus de mission permanente et, jusqu'à la fin du XVII siècle, les religieux ne viennent dans l'île depuis Sao Tomé que pour officier quelques jours par an, baptisant et mariant les habitants à la chaîne. Les ordres religieux se succèdent : à partir de 1685, les capucins sont appelés à gérer l'évêché et, vers 1691, les franciscains viennent les aider (Alencastro 2008 : 109), mais aucun d'entre eux ne vient réinstaller une mission permanente dans l'île.

L'évangélisation devenant irrégulière, les sacristains prennent une place et un pouvoir de plus en plus importants sur les insulaires. Pendant cette longue période, le souvenir du culte catholique romain et sa lecture se transforment. Les héritages africains qui ont survécu en parallèle y sont intégrés et adaptés. Cette situation particulière permet une création originale aussi bien dans les rituels que dans le culte lui-même. Si les missionnaires de passage ne se rendent pas toujours compte de ce glissement progressif des croyances et des pratiques (de Wulf 2014, t. 1 : 115-119), ce constat apparaît tout de même, de temps en temps, dans les rapports des religieux qui restent un peu plus longtemps sur place, ou qui observent plus attentivement les Annobonais ${ }^{7}$. Ces informations remontent jusqu'au pape. Ces missionnaires témoignent de leur émotion devant la ferveur religieuse des insulaires, mais se désolent de la voir « dévoyée ». Ils insistent sur la chance d'avoir des chrétiens si impliqués dans cette zone géographique, alors que dans les territoires du golfe de Guinée en général, et notamment sur le continent, il leur est souvent très difficile d'imposer le christianisme. Ils s'attristent donc devant ces « âmes » annobonaises, ces « brebis » converties et croyantes qui sont ainsi laissées « sans guide spirituel $»^{8}$.

La situation de l'évangélisation est catastrophique un peu partout dans l'empire portugais. Le Padroado manque d'efficacité et de suivi dans sa gestion. La papauté réagit face à ce constat d'échec avec la création de la Propaganda Fide en 1622 (de Alencastro 1986 : 230-231). Des missionnaires mandatés par

6. «Relatório do Bispo de Sant Tome ao Papa (24/10/1597)» (BRÁsIo 1953 : 584-585).

7. APF (Rome), Scritture riferite nei congressi (SC) : Africa, Congo, Senegal, Isole dell'Oceano Atlantico, Volume 6, fol 1-3, «Notijie estratte (S.Thomé-Anno Bono) dall Archivio dellas Congregazione de Propaganda Fide», s. d.

8. APF (Rome), SC : Africa, Congo, Senegal, Isole dell'Oceano Atlantico, Volume 6, fol. 1-3, « Notijie estratte (S.Thomé-Anno Bono) dall Archivio dellas Congregazione de Propaganda Fide », s. d. 
le pape commencent alors à venir s'installer dans l'île et tentent d'y créer une mission permanente ${ }^{9}$. Ils restent ainsi, avec quelques interruptions, environ trente ans, de 1723 à 1753. Ce sont, pour la plupart, et comme les Annobonais le réclamaient depuis longtemps déjà, des capucins italiens ${ }^{10}$.

Cette demande précise et curieuse se justifie : dès le début du XVIII ${ }^{\mathrm{e}}$ siècle, les Annobonais réussissent à se libérer du joug portugais. De fait, il semble que malgré leur isolement, ils soient très au courant de la situation géopolitique occidentale car l'Italie est alors un pays sans ambition coloniale et territoriale. Leur souhait de n'avoir affaire qu'à ces missionnaires conduit même un missionnaire français, en 1713, à se faire passer pour un capucin italien. Le frère Almaric, que les autorités portuaires de Nantes chargent de rédiger un rapport sur cette société, est alors chaleureusement reçu par les insulaires ${ }^{11}$.

Le rôle des religieux envoyés par la Propaganda Fide provoque une vive réaction des ordres missionnaires nommés par le Padroado dans le golfe de Guinée. Nombreuses sont les protestations, et ces capucins italiens seront même accusés d'être les instigateurs des "superstitions" liées au culte annobonais. Dans les faits, il semble que ces missionnaires italiens aient été mieux tolérés par la population, et se soient probablement montrés moins intrusifs que les autres. Quoiqu'il en soit, les religieux de passage qui viendront par la suite, noteront qu'un de ces capucins était vénéré par la population à l'égal d'un saint après son décès ${ }^{12}$ (de Wulf 2014, t. 1 : 130). Leur présence dure, comme précédemment évoqué, une trentaine d'années environ, mais ils ne peuvent rester plus longtemps car l'île change de statut: elle réintègre le domaine royal portugais en étant rachetée par la couronne en 1755 au capitaine donataire de l'époque. Le Padroado est, dès lors, tenu de s'en occuper à l'exclusion de toute autre entité religieuse. Le Pape n'est donc plus en mesure d'imposer ses missionnaires.

Ce changement de statut n'apporte rien aux Annobonais : les Portugais tentent à trois reprises, dans le courant $d u X \mathrm{XIII}^{\mathrm{e}}$ siècle, d'en reprendre le

9. APF (Rome), SC : Africa, Congo, Senegal, Isole dell'Oceano Atlantico, Volume 4, fol. 292 , «Relazione intorno alle missioni de S. Thomé data al V.V. Mess della Sagra Congregazione de Propaganda Fide dal P. Andrea da Mornico Padre Missionario capuccino (1723) » et APF (Rome), SC : Africa, Congo, Senegal, Isole dell'Oceano Atlantico), Volume 4, fol 319, Affrica « (P. Andrea da Mornico Cappuccino), III- Isola d'Annobuono (03/07/1724)».

10. Cette demande est récurrente dans les correspondances des missionnaires de passage, ainsi que des marins (DE AlenCAStro 1986 : 232), Frère Agustinho de Santa Maria, Santuário Mariano e história das imagens milagrosas de Nossa Senhora e das milagrosamente apparecidas..., Volume 10, 1723, p. 447, etc.

11. Archives nationales (AN) (Paris), B/3/212, fol. 234 à 236 : « colonies d'Annobón ». Lettre écrite à Nantes le 29 juillet 1713, à l'attention de M. Luzancay, Commissaire Ordonnateur - Nantes par Mancay.

12. C'est le cas du père Vincente de Brescia. 
contrôle. Parmi elles, deux sont des tentatives d'approche missionnaires : en $1757^{13}$ et en $1770^{14}$. Par deux fois, des religieux sont mandatés par le souverain portugais afin d'approcher les insulaires de la manière la plus pacifique possible, car leur intérêt pour la religion catholique lui est alors connu. Mais les Annobonais ne sont pas dupes, ils savent que l'installation dans l'île de ces missionnaires n'est que le premier pas du Portugal pour reprendre pied sur ce territoire.

Ces échecs successifs font que l'île demeure abandonnée des missionnaires et des Portugais en général. Les Annobonais peuvent donc continuer, sans entrave, à pratiquer leur culte. En plus de l'apport du catholicisme, de la survivance de rites et croyances d'origine africaine, les insulaires font preuve d'une forte capacité à créer rapidement de nouveaux rituels. Leur réactivité et adaptation leur permet d'effectuer des changements parfois étonnants dans la vie quotidienne et religieuse. Tout dépend de l'origine et de la religion des équipages qui entrent en relation avec eux. Cette particularité rend le suivi de leur évolution difficile, car la documentation aujourd'hui identifiée sur cette période est très parcellaire.

En 1777, est signé le traité d'amitié, de garantie et de commerce de San Ildefonso et, en 1778, celui du Pardo vient le compléter ${ }^{15}$. Il s'agit pour les Portugais, contre cession de territoires d'Afrique centrale et sud-américains, de délimiter à leur avantage le territoire brésilien et de récupérer leur colonie du Santo Sacramento. Après le décès du roi et le renvoi du marquis de Pombal par la reine Marie $\mathrm{I}^{\mathrm{re}}$ de Portugal, la tension entre le Portugal et 1'Espagne s'apaise. Ces traités sont l'occasion pour les Espagnols d'avoir un lieu où organiser la Traite en direction de leurs territoires américains qui nécessitent une importante population servile, notamment pour la culture de la canne à sucre à Cuba. L'Espagne a été tributaire jusque-là de l'Asiento, sorte de contrat passé avec d'autres nations européennes qui traitaient pour elle en Afrique. Ces arrangements lui coûtant très cher, et le pays commençant à être dépassé économiquement par l'Angleterre, la couronne espère pouvoir redresser son économie grâce à cette opportunité. Ces traités sont supposés lui permettre non seulement de résoudre certains litiges sur le continent sud-américain avec

13. AHU (Lisbonne), Fonds Baia, $n^{\circ} 2463$, « Termo de desobediência, e rezistencia que fizerão o Capitam mor João Dias Rapozo, moradores e povo da ilha de Anno Bom contra S. Magestade Fidelissima, e ao Reverendo Parocho Missionario da mesma ilha, Fr Francisco Pinto da Fonseca, Freyre profeço na Ordem de Christo (20/02/1757) ».

14. AHU, Fonds São Tomé, Cx 14, doc 1, « Carta de Vicente Gomes Ferreira para o SEMU (S. Antonio, 26/02/1772)».

15. AGS (Simancas), Estado 7411 bis, folio 6 (p. 4) : « Tratado de Amistad, Garantia y Comercio... (24/03/1778) » et A.G.S. (Simancas), Estado 7411, doc. 5 : Instrucción que debe observarse para proceder a tomar posesión de las islas de Annobón y Fenando Póo... (09/10/1777). 
les Portugais, mais aussi de pouvoir coopérer économiquement avec eux dans la zone du golfe de Guinée où les Lusitaniens se sont engagés à les aider à s'implanter et à développer leurs activités (de Wulf 2015a).

Mais ces estimations sont faites sans que la réalité du terrain soit prise en compte : lorsque les représentants de l'autorité royale espagnole parviennent dans le golfe de Guinée, accompagnés d'un commissaire portugais, à la fin de l'année 1778, ils s'aperçoivent que d'une part, ils n'ont pas les défenses immunitaires pour vivre sous ces latitudes et que d'autre part, les forteresses portugaises, qu'ils pensaient encore utilisables, ont disparu, et enfin que le Portugal ne contrôle, en fait, absolument rien dans ces territoires. D'ailleurs à Annobón, la population s'oppose ouvertement à ce transfert d'autorité, d'autant plus qu'elle remet en cause aussi celle du souverain portugais ${ }^{16}$. Sa façon d'exprimer son refus prend une tournure inattendue pour les Espagnols, comme pour les Portugais venus les accompagner. On y retrouve une forme de ritualisation du comportement qu'il est intéressant de mentionner ici :

Et commença une procession de femmes qui était remarquable de par son unité et ses circonstances. Elles sont venues en deux ou trois files, les unes avec des crucifix, les autres avec de grandes croix de bois, d'autres encore avec des images de saints, et la plupart d'entre elles avec des crânes et des os de défunts. En arrivant à l'église, elles en firent le tour en faisant différents gestes ou cérémonies dignes de l'ignorance et la superstition qui règnent dans cette île ${ }^{17}$.

Le jour suivant, les délégations décident d'organiser une nouvelle messe afin de tenter d'amadouer la population. Mais cette fois-ci, la foule ne les accompagne pas en procession et, une fois devant l'église, les équipages ibériques découvrent un autre aspect des croyances des Annobonais : à la porte de l'église se trouve un cercueil avec cinq crânes, un à chaque angle et l'autre au milieu, et plus loin sur une natte de palmier avec des os de défunts, entourés, semble-t-il, de lampes faites à partir de noix de $\operatorname{coco}^{18}$. Ces descriptions montrent l'influence africaine liée à un culte des ancêtres

16. A.M.N. (Madrid), MS 469, José Varela y Ulloa, « Rapport du 12/03/1779», fol. 27 verso.

17. «siguió una procesión de mugeres muy notable por el aparato y por sus circunstancias. Venían en dos o tres filas, unas con crucifixas, otras con cruces grandes de Madera, otras con imágenes de Santos, y la mayor parte con calaveras y huesos de difuntos. Al llegar a la Iglesia hireaban la rodetta con varios ademanes o ceremonias dignas de la ignorancia y superstición que reyna en aquella Isla », A.M.N. (Madrid), MS 469, José Varela y Ulloa, «Rapport du 12/03/1779», fol. 28-28 verso. Parmi ces gestes, on trouve celui de la génuflexion traditionnelle des femmes encore pratiquée aujourd'hui, la Mzuia, ou Muzura (ZAMORA LOBOCH $1962: 48$ ).

18. A.M.N. (Madrid), MS 469, José Varela y Ulloa, « Rapport du 12/03/1779», fol. 30. 
et rappelle aussi, entre-autres, le retournement des morts ${ }^{19}$. Il est possible d'en avoir également une autre lecture comme cela est, semble-t-il, le cas du commissaire portugais. Cet homme arrive du Brésil et ne supporte pas cette démonstration comme l'atteste l'équipage espagnol : «Cette vision dut effrayer le commissaire portugais, parce qu'il demanda immédiatement aux marins de son navire de transporter le cercueil à la plage, ainsi que la natte de palmier dans laquelle se trouvaient les misérables victimes de la mort $»^{20}$. Aujourd'hui, de telles pratiques ne semblent plus avoir cours, du moins ne sont-elles pas évoquées dans les écrits des Annobonais au XX⿳⺈冂大 siècle.

Suite à ce nouvel échec, rien n'est tenté pour favoriser l'évangélisation des Annobonais. Le Patronato, qui est 1'équivalent espagnol du Padroado portugais, ne remplit pas son office. Il a toutefois à sa décharge le fait qu'il n'existe que le diocèse de São Tomé dans le golfe de Guinée, et celui-ci est sous la responsabilité du Portugal. Il faudra donc attendre la création de la préfecture apostolique de Fernando Póo le 10 octobre 1855, en même temps que la nomination de son préfet, le père Miguel Manuel y Sanz par le pape Pie IX (Olanga 1943 : 20) pour que 1'Espagne commence à prendre en main l'évangélisation de ses colonies d'Afrique centrale. En attendant, les insulaires restent, une fois de plus, uniquement guidés par leurs sacristains.

Il semble pourtant que quelques tentatives santoméennes aient été menées : on trouve à la Propaganda Fide des courriers indiquant que des religieux se sont portés volontaires pour s'y rendre, mais ces demandes ne paraissent pas avoir eu de suite (de Wulf 2014, t. 1 : 284). Toutefois, les missionnaires clarétains trouvent, à la fin du XIX ${ }^{\mathrm{e}}$ siècle, des indices de ce passage : un missel portugais, datant du début du XIX ${ }^{\mathrm{e}}$ siècle, et un calendrier traditionnel établi, semble-t-il, à partir d'un modèle Pamue permettant aux insulaires de se retrouver dans le temps, mais aussi et surtout de savoir à quelle date célébrer les fêtes religieuses catholiques (Munoz y Gaviria 1871 : 221). Ce calendrier n'a qu'un défaut, il ne prend pas en compte les années bissextiles. En faisant un calcul approximatif pour déterminer la date de son introduction, on parvient là encore au début du XIX ${ }^{\mathrm{e}}$ siècle. Le premier document à le décrire est une relation de voyage de l'expédition espagnole de José de Moros y Morellon datant de 1832. Expédition d'exploration, elle n'a pas eu, contrairement à celles qui la suivront, une ambition colonisatrice (de Moros

19. Coutume pratiquée notamment à Madagascar. En résumé, elle consiste à déterrer les os de ses parents proches, à les nettoyer et à les réenterrer afin de les honorer et préserver leur mémoire.

20. «Esta visión debió de asustar al Comisario Portugués, porque mando inmediatamente a los marineros de su bote que llevaren a la playa el féretro, y la estera de palma en que estaban aquellos miserables despojos de la muerte », A.M.N. (Madrid), MS 469, José Varela y Ulloa, « Rapport du 12/03/1779 », fol. 30. 
y Morellon 1844 : 23). Les Annobonais sculptent régulièrement de nouveaux calendriers qui se présentent sous la forme d'une sorte de baguette de bois avec des encoches et des croix. Il est possible que ce calendrier soit plus ancien et que l'un de ses responsables l'ait reproduit avec une erreur, mais cela semble peu probable : les missionnaires santoméens, venus à la fin du XVIII ${ }^{\mathrm{e}}$ siècle, l'auraient certainement mentionné dans leurs écrits.

Ce n'est qu'en 1885 que la colonisation espagnole débute dans l'île, grâce à l'installation d'une mission permanente des frères clarétains du Cœur Immaculé de Marie. À partir de cette date, le culte traditionnel continua d'exister mais à l'abri des regards étrangers. Toutefois, on peut suivre son évolution dans le temps grâce à quelques événements qui le font réapparaître au grand jour. Cela est le cas, par exemple, en 1892, lors de la tentative des missionnaires de faire déménager la population aux abords de la mission pour mieux les surveiller, tout en leur proposant des logements qu'ils jugent plus sains. Cette démarche vise à amener les insulaires à abandonner l'emplacement traditionnel de leur village mais aussi une partie de leurs lieux de culte. Grâce à cette mesure, les clarétains espèrent accélérer le processus d'acculturation qu'ils ont commencé rapidement à mettre en place après leur installation. Ils sont les uniques représentants du pouvoir colonial et, après avoir observé et séduit une partie de la population par leurs enseignements, ils décident d'imposer par la force aux habitants réfractaires l'abandon du culte local et des règles de vie respectueuses de la religion catholique. Les abus sont tels que les notables de l'île font remonter leurs doléances au gouverneur général de la colonie en 1894. Besabé de la Puente décide de se rendre dans l'île en janvier 1895 et, en découvrant la situation sur place, leur donne raison. Les clarétains neutralisés, les Annobonais reprennent alors leurs pratiques traditionnelles et désertent l'église catholique (de Wulf 1997). Ce revers, très mal vécu par les missionnaires, les force à adopter une stratégie plus douce et pérenne. Ils se focalisent dès lors sur les jeunes et les femmes afin d'apporter une scission au sein des valeurs traditionnelles prônées par les hommes et les anciens et tenter de s'ancrer plus profondément dans la vie des insulaires (Creus 2015 : t. 1-2).

Ces heurts amènent le gouvernement général de la colonie à réfléchir à un meilleur encadrement des indigènes. Il en résulte notamment la création du Consejo de Vecinos en 1895 pour remplacer la « Junta de Autoridades $»^{21}$, puis celle du Patronato de Indigenas en 1904 et, enfin en 1906, la nomination d'un délégué du gouvernement colonial choisi parmi les officiers de la garde civile ${ }^{22}$.

21. AGA (Alcala de Hénares), Cx 1459, Correspondencia con las misiones (1895).

22. AGA (Alcala de Hénares), Cx 1753, n 98 : Delegación del Gobierno General en Annobón (1906). 


\section{Un culte qui se crée progressivement}

Ce n'est qu'à partir du début du XVIII ${ }^{\mathrm{e}}$ siècle, une fois le commis européen représentant le capitaine donataire dans l'île disparu, que des descriptions concernant l'organisation de la société annobonaise paraissent dans les rapports et les correspondances des voyageurs, colons, marchands ou missionnaires. On apprend ainsi qu'il existe un conseil qui dirige les habitants. Ce conseil est à la fois un héritage portugais, car proche du "Conseil des Hommes bons » qui existait dans les territoires d'outremer lusitaniens et notamment sur l'île de São Tomé, et un héritage africain, car également à l'image, par exemple, des Conseils des anciens (de Wulf 2014, t. $2: 23$ ).

Les hommes qui le constituent sont, pendant la colonisation portugaise, les notables de l'île tels le représentant du capitaine donataire, des exploitants agricoles propriétaires de lopins de terre, mais aussi des représentants religieux, lorsqu'ils sont présents. Tant que l'esclavage existe dans l'île, les esclaves sont logiquement exclus de ce conseil. Toutefois, lorsque les Portugais sont boutés hors de l'île, l'esclavage disparaissant, l'accès à ces responsabilités s'élargit. Pour faire partie de cet organe décisionnel, il faut néanmoins remplir au moins deux conditions : être un homme, car les femmes n'y sont pas admises, et être marié canoniquement (Bodipo Lisso $2015: 22$ ). En effet, un homme n'est considéré adulte qu'une fois son mariage religieux célébré $^{23}$. Le conseil n'est donc constitué que par des hommes jugés sur leur statut comme étant adultes et responsables.

Les représentants religieux du culte annobonais sont les sacristains. Leur origine laïque leur permet de conserver le droit, et même le devoir, d'être marié. Ils siègent donc, à ce titre, au Viyil qui est le nom du conseil. À leur tête se trouve le sacristain majeur (Zamora Loboch 1962:25). Il a à sa charge l'église principale de l'île et préside le conseil aux côtés du gouverneur (ou capitão-mor) ${ }^{24}$ (de Wulf 1997 : 24). Chacun des autres sacristains est responsable d'une chapelle. Les missionnaires clarétains au début du XXe siècle, aidés parfois du délégué du gouvernement colonial, en détruisent une grande partie (de Wulf 2014, t. 2 : 128). Malgré ces actions violentes, leurs emplacements restent, aujourd'hui encore, symbolisés par une croix et des rituels s'y déroulent toujours. Les chapelles sont dédiées à des saints. Le sacristain d'une chapelle a le devoir de prendre soin des statues et/ou des ornements qui y sont conservés.

23. Il existe parallèlement un mariage dit « coutumier » qui n'a pas de valeur pour un homme souhaitant devenir un des notables de l'île. La religion joue donc un rôle de filtre dès le début de la création de cette société.

24. L'église du sacristain majeur est incendiée peu de temps après l'installation de la mission clarétaine à la fin du $\mathrm{XIX}^{\mathrm{e}}$ siècle et n'a pu être reconstruite depuis. 
Certains de ces trésors ont été « sauvés » de la destruction et continuent d'être sortis lors des jours de fête de ces saints pour une procession ou pour la célébration d'une messe traditionnelle locale (Barrena $1965: 18$ ).

Les femmes ont un rôle limité dans ce culte, à l'exception des veuves devenues beatas qui acceptent la chasteté à vie, et prient à la fois pour leur défunt mari et pour la communauté tout entière. Ces « saintes » sont autorisées à aider le sacristain majeur dans son office et lors de certaines célébrations (de Wulf 2014, t. 2 : 108-113). Une beata peut cumuler son statut avec celui de soñadora, c'est-à-dire de « rêveuse ». Il s'agit en fait d'une voyante, mais la sorcellerie étant fortement redoutée dans l'île, une rêveuse ne peut avoir de vision qu'en dormant. La transe n'existe donc pas officiellement dans la société annobonaise (ibid. : 114-115). Tout comportement inhabituel des femmes âgées est d'ailleurs très mal perçu : c'est ainsi que les femmes ménopausées, victimes de bouffées de chaleur, qui sont aperçues en train de prendre un bain de mer la nuit, sont considérées d'office comme des sorcières. Elles sont supposées copuler avec le diable ${ }^{25}$. Aucune solution de rédemption ne leur est offerte. Poursuivies par des milices qui se créent pour l'occasion, elles sont lapidées, avant la venue des missionnaires santoméens en 1770: «Les dits chanoines ont sauvé de la mort quatre femmes qu'ils [les gens d'Ano Bom] voulaient tuer comme sorcières, et ce fut le motif du premier soulèvement du 8 novembre $»^{26}$. Cette peine est commuée, dès lors, en abandon en haute mer à bord de pirogues avec seulement quelques vivres et de l'eau (Zamora Loboch 1962:61). Au début du XIX ${ }^{\mathrm{e}}$ siècle, les plus chanceuses ont rejoint l'île de São Tomé et constituent une petite communauté d'une cinquantaine de personnes (da Cunha Matos 1963 : 113-114). Devenir beata permet, $a$ priori, de ne pas entrer dans la catégorie de ces femmes potentiellement sorcières (Zamora Loboch 1962 : 62). Elles ont un privilège attesté là encore en 1770 : «Toutes les veuves vont chaque samedi vers midi à l'église avec leurs ragoûts dans des marmites pour dîner avec Notre-Dame, car elles sont des Saintes ${ }^{27}$. Ce repas devait sans doute se passer après un office religieux. Une fois le repas achevé, il est rapporté qu'elles se livrent à des danses dans le lieu saint. Le témoin de cette cérémonie, visiblement horrifié, les qualifie

25. Conférence du 21 juillet 2011 au centre culturel espagnol de Bata (Guinée équatoriale), sur le thème de «L'influence de la sorcellerie dans la société équato-guinéenne », intervention du professeur José Fenando Siales.

26. « Os dittos Conegos livrarão da morte a quatro mulheres, que elles querião mattar por feiticeras, que foi o motivo do primeiro levante de oito de Novembro ", extrait de A.H.U., Fonds São Tomé, Cx 13, doc 15, « Sobre os costumes e tradições de Ano Bom (20/12/1770) ».

27. «Hirem todas veuvas todos os Sabbados à Igreja pelo meio dia com panelas de seus guizados a jantar com Nossa Senhora, por rezao de serem beatas. » A.H.U. (Lisbonne), Fond São Tomé, cx13, doc 15, « Sobre os costumes e tradiçoes de Ano Bom (20/12/1770) ». 
de « malhonnêtes $»^{28}$, alors que compte tenu du contexte, il ne peut s'agir que de danses à caractère religieux, expression de la foi de ses femmes, en forme d'hommage rendu à la Vierge. Il était d'ailleurs de mise de danser pendant l'office religieux en Europe jusqu'au XVIII siècle. Les danses étaient exécutées à trois moments différents de la célébration, élément qui a pu séduire les Annobonais très attachés à ce chiffre ${ }^{29}$.

Le rôle du culte local dans la vie des insulaires

C'est le culte traditionnel qui constitue la trame de cette communauté. Les dignitaires religieux ont de grands privilèges et sont consultés sur tous les aspects de la vie des insulaires. Ce sont eux qui, en grande partie, rendent possible la cohésion de ce peuple grâce à leurs conseils avisés (de Wulf 2014, t. 2 : 92-97). L'influence de ce culte se retrouve donc à tous les niveaux de cette société : dans la vie courante des habitants, aussi bien que dans les moments exceptionnels.

Les sacrements forment les différents niveaux initiatiques de cette culture à l'image des sociétés chrétiennes occidentales : tout commence avec le baptême, cela se poursuit avec le mariage, le veuvage pour s'achever en partie avec l'extrême onction et la fête des morts. Chaque étape de la vie sur terre prépare donc à la vie dans l'au-delà : le baptême permet un enterrement digne aux enfants et l'assurance que leur âme ira au paradis sans rester errer sur Terre. Il semble que les âmes des non-baptisés soient maintenues, ou peut-être calmées, par un rituel qui se pratique autour de croix. En effet, les enfants sont souvent enterrés dans la rue ou dans les maisons aux environs des nombreuses croix qui protègent les cases des habitants (Barrena 1965: 32). Ce rituel est décrit par un missionnaire clarétain à la fin du XIX ${ }^{e}$ siècle. Ceux qui sont baptisés sont en général les plus âgés des habitants ou ceux qui étaient là lors du passage de missionnaires. Ce cap franchi, l'intégration des enfants dans la société peut alors commencer par l'intermédiaire de confréries religieuses et sociales. Héritage à la fois portugais et africain, ces sociétés d'entraide se présentent sous deux formes principales : les confréries de classe d'âge, à l'intérieur desquelles la journée et la semaine de naissance ont une importance, et les confréries de $\operatorname{sex}^{30}$. Ces confréries accompagnent

28. A.H.U. (Lisbonne), Fond São Tomé, Cx 14, doc 1, «Carta do Vicente Gomez Ferreira $(26 / 02 / 1772) »$.

29. Certaines prières ou cantiques sont souvent répétés trois fois, ce chiffre semble très important dans les rituels annobonais.

30. On trouve ces composantes dans de nombreuses sociétés africaines, mais aussi, pour une part, dans la société portugaise. 
l'individu tout au long de sa vie, lors des événements heureux ou malheureux qu'il rencontrera, mais surtout lors des cérémonies religieuses qui jalonnent son parcours (de Wulf 2014, t. 2 : 163-169).

Lorsqu'un mariage est célébré, il dure une semaine environ et est accompagné de plusieurs messes et rituels qui vont se dérouler dans différents lieux emblématiques de l'île. Un des plus importants se trouve au bord de la rivière San Juan au lieu-dit A-bobo. Il s'agit d'un des endroits où les âmes des défunts se rendent en attendant l'âme de leur conjoint pour pouvoir se présenter en couple devant le « Tribunal Suprême» avant d'aller au Paradis. Tous les participants à la noce y déjeunent le dernier jour des festivités. À cette occasion, un peu de nourriture est mise de côté pour les défunts. Il s'agit le plus souvent de restes éparpillés car le repas dure plusieurs heures. Ce moment exceptionnel lié à ce lieu consacré est l'une des rares occasions où vivants et morts sont supposés être réunis afin de valider ensemble cette union, ce qui représente l'une des expressions de la survivance du culte des ancêtres dans le monde religieux annobonais ${ }^{31}$. C'est aussi lors de cet événement que, dans l'onde de la rivière, les vêtements des mariés sont nettoyés par leurs proches $^{32}$. Ce rite magique scelle ainsi le destin religieux du couple dans le mariage. Le manquement à ce rituel est vécu par la plupart des insulaires comme une catastrophe : il sera très difficile, voire impossible, aux deux âmes de se retrouver le jour venu.

Le veuvage est l'étape intermédiaire suivante. De peu de conséquence pour l'homme, car il a toujours pu au-delà d'une année à nouveau convoler, il a été en revanche longtemps beaucoup plus lourd pour les femmes puisqu'il leur était impossible de se remarier sous peine d'être considérées comme adultères. Toutefois, grâce à l'influence des clarétains, devenir beata en faisant vœux de chasteté n'est plus aujourd'hui une obligation, les veuves peuvent elles aussi se remarier si elles le souhaitent.

L'extrême onction figure parmi les dernières étapes de la vie d'un insulaire mais constitue aussi l'occasion de rituels collectifs : tout d'abord la famille du mourant ne le laisse jamais seul. Il bénéficie non seulement du soutien moral de ses proches, mais également de soutien physique car la personne qui le veille le tient dans ses bras ou la tête posée sur ses genoux (Fernandez

31. Ce qui est inhabituel dans de nombreuses ethnies africaines, car on tente d'éviter que les morts ne puissent venir hanter les vivants comme nous l'évoquerons plus loin.

32. Comme M. Zamora Loboch (1962) et P. Bodipo Lisso (2015) n'écrivent pas la même chose sur ce sujet, j'en déduis que les années qui séparent leurs témoignages ont été marquées par de nombreux changements, et notamment l'embauche d'Annobonaises sous-payées pour des cérémonies de ce type, organisées par des insulaires aisés aux revenus provenant de Malabo ou du continent africain. Nous sommes alors bien loin de la période qui nous intéresse (entretiens de 1996 et 2011). 
1962 : 183). Si l'agonisant est marié canoniquement, c'est son conjoint qui l'accompagne lors de la confession de ses pêchés et jusqu'à son décès. On lui maintient alors les mains jointes et on lui place un cierge qui restera allumé jusqu'à son dernier souffle (Zamora Loboch 1962 : 53). Pendant ce temps, dans les rues du village, sont dites des prières, sont chantés des cantiques, et les femmes, notamment celles de la confrérie dont le mourant fait partie, pleurent et crient (Bodipo Lisso 2015 : 27). Selon les croyances locales, il y a une vie après la mort. L'âme chemine à travers l'île avant de faire le tour du monde et de revenir. Elle a la capacité de se nourrir comme un être vivant. Les dons de nourriture sont donc importants, surtout à l'époque de la fête des morts. Nombreuses sont alors les veuves qui se privent de nourriture au profit de leur défunt mari (Zamora Loboch 1962 : 55-57).

Un rite initiatique récupéré par le culte local: la chasse à la baleine

À la fin du mois de mai, ou au début de juin, des baleines et leurs baleineaux croisent dans les eaux annobonaises. C'est l'occasion pour les jeunes gens de montrer leur bravoure et de faire un geste pour la communauté. Cette tradition est très forte car elle est représentative de la grande solidarité qui existe entre les habitants. Elle est aussi extrêmement codifiée. Tout d'abord, ce sont les soñadoras (les voyantes) qui désignent le jeune homme qui aura le plus de chance de réussir à capturer un baleineau. Les ancêtres leur soufflent le nom de l'heureux élu. Grâce à cette manifestation des défunts, cet événement se trouve récupéré par le culte local et fait ainsi corps avec le reste des croyances et des rites qui ponctuent la vie des insulaires. Après ce premier choix décisif, le reste est géré par les individus eux-mêmes : c'est au pêcheur désigné de choisir un équipage limité à trois personnes au total. Les autres pêcheurs les accompagnent à bord de leurs propres pirogues. Ils ne peuvent leur prêter main forte, en principe, qu'une fois le baleineau séparé de sa mère et harponné. Ils sont alors autorisés à aider à son immobilisation en le harponnant à leur tour, et en s'accrochant les uns derrière les autres à la corde du premier arpon afin d'épuiser l'animal au plus vite. Une fois celui-ci à bout de force et sa mère déboutée, l'animal est attaché à la pirogue du pêcheur élu et ramené jusqu'à la plage. Dès qu'il est hissé sur le sable, commence une grande distribution au profit de tous les insulaires. Seules quelques pièces particulières, comme la tête, reviennent au pêcheur désigné et à son équipage. Le pêcheur va alors offrir sa part de trophée à ses parents (Zamora Loboch 1962 : 68-72). Il y a dans cette épreuve une dimension initiatique, mais elle n'est plus offerte aujourd'hui qu'à quelques élus, du fait de la raréfaction de ce cétacé dans 
les eaux insulaires. Les pêcheurs ayant réussi ce tour de force restent dans la mémoire de tous les habitants : le jour de leur enterrement, leur pêche exceptionnelle est traditionnellement mimée dans le cortège accompagnant leur corps au cimetière, afin de rendre un dernier hommage à leur courage et à leur habileté.

\section{Le rôle protecteur du culte annobonais}

Les insulaires honorent Dieu de différentes manières, notamment avec le chant et la danse : de nombreux cantiques sont composés, et ce par tous les habitants ayant des talents de paroliers et/ou de compositeurs. Ils sont ensuite repris et dansés lors de fêtes religieuses, notamment par les fessa, les membres de la confrérie des femmes, avant leur mariage. Par cet acte de dévotion, ces jeunes femmes contribuent, à leur manière, à l'effort de protection physique et spirituelle que le culte traditionnel a mis en place afin de protéger les insulaires des malheurs. Ce genre d'initiatives artistiques nous permet de mieux comprendre comment de nombreux rites ont vu le jour dans le but d'anticiper les ennuis auxquels les insulaires pouvaient être confrontés.

Certains des rituels supposent la participation de tous les habitants. La notion de partage et de solidarité étant très importante, c'est autour de ces célébrations que la population se fédère pour un meilleur avenir. Par exemple, lors de la fête de la Saint-Jean, tous les participants, après la messe, sautent au-dessus d'un grand feu et vont se baigner dans la mer. Les habitants sont ainsi supposés être tenus à l'écart des maladies pour une année (Bodipo Lisso 2015 : 33).

L'apport animiste et l'attachement à l'île apparaissent fortement dans ce culte à travers notamment deux cérémonies. La première vise à empêcher les hommes, tous des pêcheurs, de disparaître en mer. Le sacristain majeur et les beatas organisent une messe et des offrandes au roi de la mer (Zamora Loboch 1962 : 31-32). Très codifié, ce rite met, comme toujours, en avant les représentants de son clergé, mais les femmes y sont tout de même en première ligne : les rêveuses, les soñadoras, en fixent la date, et les veuves ainsi que les beatas rassemblent ensuite les offrandes faites au roi de la mer. Le relai est ensuite passé aux hommes de la société une fois la messe et la procession achevées : il leur faut partir en haute mer, espace réservé aux hommes, y verser les offrandes à une heure très précise et revenir très vite sur l'île en ne regardant surtout pas en arrière, sous risque de se faire avaler avec les offrandes.

Pour prévenir les risques de sécheresse, le sacristain majeur organise un rituel en l'honneur de la reine du lac. Il s'agit de lui offrir, en plus d'une messe, le nettoyage des rives de son lac d'eau douce qui se trouve dans les 
hauts de l'île. Grâce à cette célébration, certaines maladies peuvent être ainsi évitées une partie de l'année (Bodipo Lisso 2015 : 18).

Les Annobonais redoutent beaucoup le diable. Il hante les histoires et les contes (Barrena 1965: 40). Il a longtemps ressemblé à un homme blanc (de Wulf 2014, t. 2 : 192). Ses représentations actuelles lui attribuent essentiellement les couleurs rouge et/ou noir suite à des influences culturelles étrangères récentes ${ }^{33}$. Il arrive qu'il prenne possession d'un homme ou d'une jeune femme. Dans ce cas, le sacristain majeur isole la personne en question et lui administre, pendant plus d'une semaine, un traitement constitué surtout de nombreuses prières (Bodipo Lisso $2015:$ 19).

Pour lutter contre les sorts lancés par des individus malfaisants, il existe toute une série de rites en fonction de la gravité des malheurs que les habitants peuvent connaître. Ces contre-offensives ou parades sont officiées par les sacristains. Le plus redoutable d'entre eux est le Mete Cusu Awa (d'Al), il suppose l'action synchronisée de tous les sacristains en divers lieux de l'île au moment du lever du soleil (Bodipo Lisso 2015 : 31).

Avec la venue des missionnaires clarétains, puis des colons espagnols, s'est mis en place un trafic maritime entre l'île et le reste de la Guinée espagnole insulaire et continentale. Les Annobonais sont allés travailler en dehors de leur île et ont été influencés par d'autres cultures. Ils ont ainsi ramené des masques qu'ils ont adaptés à leurs croyances et intégrés à leur culte. Parmi ces nombreux masques, le pilate est censé représenter Ponce Pilate et a un rôle curatif préventif (ibid. : 41). Il n'est pas le seul à avoir un rôle curatif: lorsque quelqu'un a une maladie avérée, ou que se prépare un accouchement, le sacristain majeur vient apporter sa bénédiction (ibid. : 18). C'est parfois le luto, un masque revêtu d'un costume noir à tête de mort et à deux tibias croisés blancs cousus dessus, qui s'en charge. Ce masque est lui aussi une création récente ${ }^{34}$. Il lui arrive également de sortir la nuit lors de la veillée de funérailles ou de la fête des morts, son rôle est alors d'aider les âmes des défunts qui ont du mal à quitter leur enveloppe charnelle ou qui errent encore (ibid. : 35).

33. C'est le cas, par exemple, du masque qui se nomme debul qui est censé le représenter, il s'agit d'une création récente du milieu du $\mathrm{XX}^{\mathrm{e}}$ siècle selon Pedro Bodipo Lisso (entretien de 1996).

34. Entretien de 1996 avec Pedro Bodipo Lisso. 
Nombreuses sont les autres pratiques syncrétiques du culte annobonais. On en retrouve cependant distinctement la trame centrale d'influence catholique sur laquelle d'autres composantes sont venues se greffer avec le temps et en fonction des besoins de la population (sécheresse, catastrophe naturelle ou humaine, etc.).

Une autre question demeure : pourquoi les habitants de l'île d'Annobón ont-ils développé une si grande religiosité ? La perte de valeurs africaines au contact des Santoméens avant leur installation à Annobón y a-t-elle contribué ? À cela, il faut probablement ajouter le sentiment continu du danger et de leur vulnérabilité sans arme pour se défendre, surtout à partir du XVIII ${ }^{\mathrm{e}}$ siècle. De ce fait, ne s'agirait-il pas aussi d'une stratégie de survie face aux Occidentaux afin de se placer sous la protection du Pape?

En tous les cas, grâce à lui, les Annobonais ont non seulement survécu, mais ils sont aussi parvenus à se créer une identité originale et forte. Ceux qui ont été forcés de quitter l'île afin de subvenir aux besoins de leur famille (à partir $\mathrm{du} \mathrm{XX}^{\mathrm{e}}$ siècle principalement) ont ainsi perpétué leurs traditions et leurs rituels malgré l'éloignement. La plupart, contraints de vivre loin de leur île, espèrent d'ailleurs y retourner pour un séjour ou pour y finir leurs jours, une fois à la retraite. Leur quête de retrouvailles familiales et de retour aux sources s'accompagne d'une sorte de pèlerinage, car ils privilégient la visite de certains sites de l'île qui n'ont, a priori, rien de touristique, tel le lieu-dit A-bobo. Ainsi, les liens qui unissent Annobón à ses habitants et leurs descendants restent forts ${ }^{35}$.

De nombreuses questions se posent encore dans le descriptif et la compréhension de ce culte que les seuls documents écrits ne suffisent pas à éclaircir. Compte tenu des difficultés de se rendre sur place et d'y mener des enquêtes, je pense élargir mes recherches à la diaspora vivant en dehors de l'île. Je sais que l'influence de l'Église évangéliste est très forte à Malabo parmi les Annobonais, et il serait intéressant d'en mesurer l'impact sur les rituels traditionnels. Par ailleurs, il est difficile en tant que femme étrangère de se renseigner auprès des sacristains. Ils aiment conserver leur anonymat puisqu'ils pratiquent le plus souvent en dehors de la présence des clarétains, et ne se réunissent qu'entre hommes. Toutefois, ces dernières années, étant en contact avec un nombre croissant d'Annobonais intéressés par mes recherches, certaines de celles-ci s'en trouvent facilitées. Les insulaires sont manifestement sensibles au fait que l'on s'intéresse à leur culture, leur histoire et leur culte, comme ils sont attachés à l'écriture et à la préservation de leur patrimoine culturel. J'espère que non seulement j'aurai l'occasion de lire d'autres écrits, que je pourrai mener d'autres enquêtes de terrain, mais surtout que je pourrai

35. Entretiens, 2011. 
mettre en place un petit groupe de recherche équato-guinéen qui pourra suivre au quotidien l'évolution rapide de ce culte. Le partage et l'échange sur un terrain difficile comme celui-ci me semble, à ce jour, indispensable.

Centre de recherches sur les mondes Américains (CERMA- EHESS, Paris); Centro de Estudios Afro-Hispánicos de la UNED (CEAH-Madrid).

\section{BIBLIOGRAPHIE}

DE Alencastro L. F., 1986, Le Commerce des vivants : traite d'esclaves et "Pax Lusitana" dans l'Atlantique Sud, Thèse de doctorat, Nanterre, Université Paris X.

— 2006, O Trato dos Viventes, formação do Brasil no Atlântico Sul, São Paulo, Ed. Schwarcz, $4^{\mathrm{e}}$ éd.

— (DIR.), 2004, « La cogestion lusitanienne et luso-brésilienne de l'empire portugais dans l'Atlantique Sud », in F. BETHENCOURT (dir.), L'Empire portugais face aux autres empires, Paris, Maisonneuve \& Larose-Centre Calouste Gulbenkian.

Ambrosio A., 1987, « Dona Simoa de São Tomé em Lisboa - O seu testamento e a sua capela », Lisboa, Revista municipal, $21: 3-22 ; 22: 25-40$.

Bosman G., 1705, Voyage de Guinée ..., Londres, Mortier (« Vingtième lettre ») : 442-445.

Barrena N., 1965, La Isla de Annobón, Barcelona, Publicaciones del Instituto Claretiano de Africanistas.

Bodipo Lisso P., 2015, Annobón : su tradición, usos y costumbres, Paris, Association France-Guinée équatoriale-L'Harmattan.

BrÁsıo A., 1953, Monumenta Missionaria Africana, Vol. III, Africa Occidental, Lisboa, Agência Geral do Ultramar.

CAldeira A. M., 2007, « Tráfico de escravos e conflitualidade : o arquipélago de São Tomé e Princípe e o reino do Congo durante o século XVI », Actas da Mesa Redonda Internacional sobre Mbanza-Kongo, Angola.

CORTESÃo A., 1971, Descobrimento e cartografia das ilhas de S. Tomé e Princípe, Coimbra, Junta de Investigações do Ultramar.

Creus J., 2015, Action missionnaire en Guinée Equatoriale, 1858-1910, t. 1, Mémoire et naïveté de l'Empire, t. 2, À la reconquête de l'Ancien Régime, Paris, L'HarmattanAssociation France-Guinée équatoriale. 
Da Cunha Matos, R. J., 1963, Compendio histórico das possessoes de Portugal na África, Rio de Janeiro, Ministério da Justicia e Negocios Interiores, Arquivo Nacional.

FERNANDEZ C., 1962, Misiones y misioneros en La Guinea española (1883-1912), Madrid, Ed. Coculsa.

Lourenço Farinha A., 1942, A expansão da Fé na Africa e no Brasil, Lisboa, Divisão de publicações e biblioteca, Agencia Geral das Colonias.

De Moros y Morellon J., 1844, Memoria sobre las islas de Fernando Póo y Annobón, Madrid, Compañia tipográfica.

Muñoz y Gaviria J. Vizconde de San Javier, 1871, Tres años en Fernando Póo : viaje a África, Madrid, Urbano Manini.

Das Neves C. A., 1989, São Tomé e Princípe na Segunda Metado do Século XVIII, Madeire, Centro de Estudos de Historia do Atlantico.

Olanga A, 1943, «El Dr D. Miguel Martinez y Sanz, primer Prefecto Apostólico de Fernando Póo y demas islas del Golfo de Guinea », Africa, ano II, $15: 20$.

Pujadas T. L., 1968, La Iglesia en la Guinea Ecuatorial - Fernando Póo, Madrid, Imp. Iris de Paz.

DE Wulf V., 1997, «Une étape dans la stratégie missionnaire clarétaine : le déplacement du village principal d'Annobón, Guinée Equatoriale (1892-1895) », Studia Africana, $8: 21-34$.

—2014, t. 1, Histoire de l'île d'Annobon et de ses habitants du XVe-au XIXe siècle (Guinée équatoriale), t. 2, Les Annobonais, un peuple africain original (Guinée Equatoriale, XVIII ${ }^{e}$ $X X^{e}$ siècle), Paris, éd. Association France-Guinée équatoriale-L’Harmattan (« Guinée équatoriale $»)$.

— 2015a, «L'acte de naissance d la Guinée espagnole : les traités d'Ildefonso, du Pardo, l'expédition du comte d'Argelejos et ses conséquences (1777-1785)», Revue GERAHA, 1 : 51-74.

— 2015b, «Le voyage du frère Almaric à l'île d'Annobón », in J. C. BoIXADERAS, J.-M. Lefebvre, G. Nerin, E. Pijning \& V. DE Wulf (dir.), La Guinée équatoriale aux Archives nationales (XVIII'-début XXe siècles), Paris, L'Harmattan-Association France-Guinée Equatoriale : 9-31.

ZAmora Loboch M., 1962, La Noticia de Annobón (su Geografia, Historia y Costumbres), Madrid, Publicaciones de la Diputación Provincial de Fernando Póo. 
RÉSUMÉ

Mener une enquête de terrain en Guinée équatoriale est une tâche complexe, coûteuse et incertaine. Mais se pencher sur l'aspect historique du culte annobonais permet d'en éclairer le présent même si les rituels évoluent très vite. Partager cet article et les connaissances acquises est l'occasion d'ouvrir un dialogue avec d'autres chercheurs mais aussi des Annobonais qui s'y intéressent. Car c'est aussi grâce à des témoignages écrits par des insulaires qu'il a été possible de reconstituer partiellement l'évolution de ce culte entre le XVe et le XXe siècle. De ce culte, il apparaît aujourd'hui qu'il a fortement contribué à rapprocher la population, grâce notamment à ses confréries. Il accompagne les insulaires de façon très codifiée aux différentes étapes de leur vie et au-delà, grâce à un culte des ancêtres. Il a aussi ancré la population géographiquement en attribuant à différents lieux de l'île une fonction bien définie.

Mots-clés: Afrique, Guinée équatoriale, culte, enquêtes de terrain, histoire, rituels, syncrétisme.

ABSTRACT

Research in Difficult Terrain: The Case of Traditional Annobonese Worship (Annobon Island, Equatorial Guinea, 15th-20th century). - Conducting a field survey in Equatorial Guinea is a complex, expensive and uncertain task. Fortunately, to look at the historical aspect of the Annobonese cult allows for shedding light on the present even if the rituals evolve very quickly. Sharing this article and the knowledge gained is an opportunity to open a dialogue not only with other researchers, but also with interested Annobonese. Testimonies written by islanders aided greatly in reconstituting the evolution of this cult between the 15th and the 20th centuries. It appears today this cult has greatly contributed to bringing the population closer, in particular its brotherhoods. The cult accompanies islanders in a very codified way at different stages of their life and beyond, thanks to ancestor worship. It has also anchored the population geographically by giving different places of the island well-defined functions.

Keywords: Africa, Equatorial Guinea, field surveys, history, rituals, syncretism, worship. 